\title{
Original
}

\section{Bone Restoration Ability of a Composite Consisting of $\beta$-Tricalcium Phosphate and Carboxymethyl-Chitin in Canine Mandible}

\author{
Daisuke Yamauchi' ${ }^{1}$, Takao Watanabe ${ }^{1)}$, Kazuhiko Nishio(1), Yusuke Yoshihara ${ }^{2}$ and Tsuneo Takahashi ${ }^{1)}$ \\ 1) Department of Anatomy, Kanagawa Dental College, Yokosuka, Japan \\ 2) Research Department, Kyocera Medical Corporation, Osaka, Japan \\ (Accepted for publication, September 19, 2013)
}

\begin{abstract}
TCP is a biodegradable bone regenerative material which has been noted to possess several limitations due to its granular property. Similarly, CM-chitin is a sponge-like material known to decompose in the body.Therefore, in order to improve the use of $\beta$-TCP, the combined form of $\beta$-TCP/CM-chitin was developed and its bone regenerative properties were tested. This study consisted of four test groups: The empty control group, the hydroxyapatite group, the $\beta$-TCP granule group, and the $\beta-\mathrm{TCP} / \mathrm{CM}$-chitin group. These materials were implanted in the mandible of canines and bone samples were harvested at 2 weeks, 1 month, and 3 months after implantation.Histological sections were prepared with hematoxylin-eosin staining. Lastly, degrees of new bone formation in the bone defects were measured by image analysis software. Although the empty control group had a smaller quantity of new bone compared with other groups at 1 month, the empty control group became similar to the latter two groups at 3 months. The HA group was similar to the $\beta$-TCP group and the $\beta$ $\mathrm{TCP} / \mathrm{CM}$-chitin group at 1 month, however the HA group was less than the other groups after 3 months. Finally, the quantity of new bone in the $\beta$-TCP group and the $\beta$-TCP/CM-chitin group increased faster and more sufficiently than in the other groups at all stages. No statistically significant differences were recognized in each group at each stage. Although smaller amounts of $\beta$-TCP granules were used in the $\beta$-TCP/CM-chitin group, this group showed the same amount of bone formation as the $\beta$-TCP group, thus suggesting that CMchitin does not inhibit bone growth. Furthermore, due to CM-chitin's sponge-like structure, the $\beta$-TCP/CMchitin group was easier to handle than the $\beta$-TCP granules alone. These observations suggest that $\beta$-TCP/CMchitin is a useful bone substitute and may have applications to oral surgery.
\end{abstract}

Keywords: Beta-tricalcium phosphate ( $\beta$-TCP), Bone substitute, Carboxymethyl-chitin (CM-chitin), Guided bone regeneration, Mandible

\section{Introduction}

$\beta$-tricalcium phosphate $(\beta$-TCP) is a highly absorptive bone regenerative material which biodegrades over time ${ }^{1)}$. Clinically, $\beta$-TCP has already been widely used as a bone substitute, and thus has been proven to be a safe and favorable bone regenerative material ${ }^{2-5}$. In animal studies involving $\beta$-TCP, histological evaluations showed its tissue compatibility to be superior to other synthetic materials as the bone substitute is resorbed and replaced by normal bone when implanted in an area of bone defect ${ }^{6}$. In order to have a better understanding, in recent times, research has been done to study the molecular mechanisms behind bone formation by $\beta$-TCP as well ${ }^{6-10)}$.

However, there are several limitations to $\beta$-TCP due to its granular property. Not only is it difficult to transport and measure accurately, but when using $\beta$-TCP in large bone defects or bleeding sites, these granules easily flow out. Furthermore, overfilling of

Correspondence to: Dr. Tsuneo Takahashi,Department of Anatomy, Kanagawa Dental College, 82 Inaoka-cho, Yokosuka-shi, Kanagawa, 2388580 Japan; Phone: +81-46-822-8845; Fax:+81-46-822-8729; Email:t.takahashi@kdu.ac.jp granules may encourage the suppression of cell infiltration to the central part of bone defects in the initial stage.

In order to solve these problems, $\beta$-TCP/CM-chitin was used to aid in bone regeneration. $\beta$-TCP/CM-chitin consists of $\beta$-TCP granules and a sponge-like water-soluble polysaccharide called carboxymethyl-chitin (CM-chitin; degree of carboxymethylation $70-80 \%$, degree of deacetylation $20-30 \%$ ). $\beta$-TCP granules were embedded in the frame of CM-chitin's three-dimensional spongy structure. A water-soluble derivative of $\mathrm{N}$-acetyl-D-glucosamine ${ }^{11)}$, CM-chitin is known to be enzymatically decomposed by lysozyme and N-acetyl-glucosaminidase with bacteriolytic activity produced by inflammatory cells, such as monocytes and neutropils ${ }^{12}$. Therefore, CM-chitin is known to rapidly decompose, excrete, and metabolize in the body ${ }^{12)}$. CM-chitin is a biologically safe material as it has neither genetic toxicity nor carcinogenicity ${ }^{20}$ ) and thus has been widely used in tissue engineering ${ }^{14,15)}$. Chitin also has been found to promote faster wound healing as it enhances immunological, antibacterial, and drug delivery activities in the body $^{15,16)}$. 


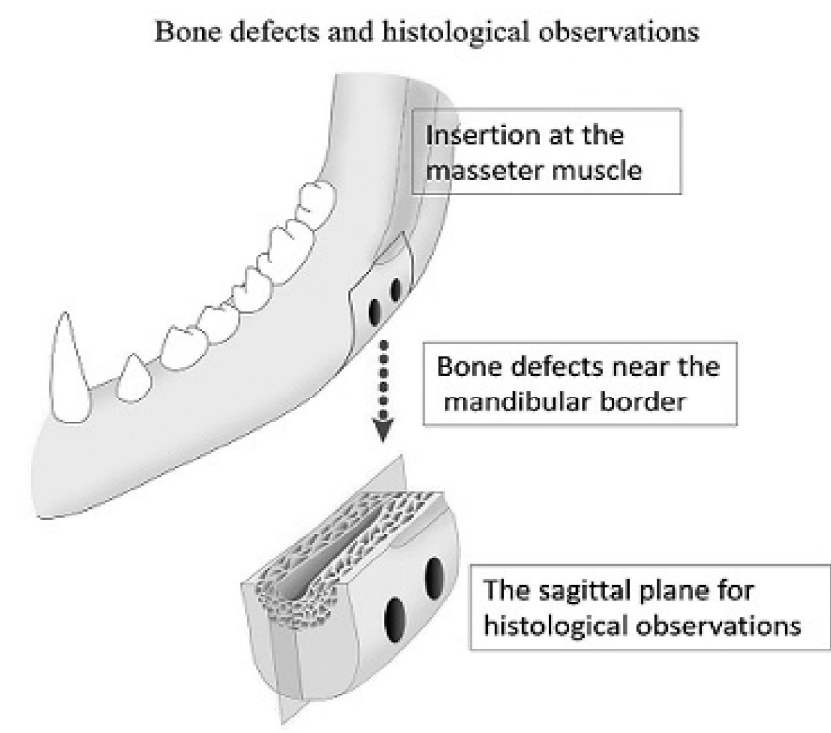

Figure 1. Two bone defects were prepared near the insertion point of the masseter of the left mandible at which $\beta$-TCP/CM-chitin was implanted into the posterior defect, and hydroxyapatite material was implanted in the anterior defect. Two similar defects were prepared on the right mandible at which $\beta$-TCP granules were implanted into the posterior, and no materials were implanted into the anterior defect. Bone blocks with the defects were harvested for histological investigation and were cut at the sagittal plane of the mandible.

Several animal studies have confirmed the effect of $\beta$-TCP/ CM-chitin to promote new bone formation ${ }^{17,18)}$, but few has been conducted in the oral cavity. The aim of this study is to observe bone restoration ability of a bone substitute consisting of $\beta$ tricalcium phosphate and carboxymethyl-chitin in the mandible of canines in order to use this material in oral surgery

\section{Materials and Methods}

\section{Preparation of test pieces}

The solution of CM-chitin which is a water-soluble derivative of chitin and $\beta$-TCP granules (particle size $50 \mathrm{~mm}-150 \mathrm{~mm}$ ) was mixed, and $\beta$-TCP/CM-chitin test pieces (diameter $\mathrm{f} 4 \mathrm{~mm}$ length $10 \mathrm{~mm}$, Japan Medical Materials Corporation, Japan) were prepared through freeze-drying and heat-treating techniques at $140-160^{\circ} \mathrm{C}$ in a vacuum. The $\beta-\mathrm{TCP} / \mathrm{CM}$-chitin test pieces were then sterilized by $25 \mathrm{kGy}$ gamma ray irradiation. Similarly, $\beta$ TCP granules (particle size $550 \mathrm{~mm}-150 \mathrm{~mm}$, Taihei Chemicals Limited, Japan) were prepared after sintering under $1,100^{\circ} \mathrm{C} 2$ hours followed by sterilization by $25 \mathrm{kGy}$ gamma ray irradiation.

\section{Animal experiment}

Sixteen mature female beagles (9-14 kg in weight) were used. All animals were handled in accordance with the Guidelines for Animal Experiment Ethics Committee of Kanagawa Dental College. Ketamine hydrochloride $(0.2 \mathrm{ml} / \mathrm{kg}$ weight $)$ was injected and submucous infiltration anesthesia ( $2 \%$ xylocaine) was administered in the oral cavity. The mucosa of the ascending branch

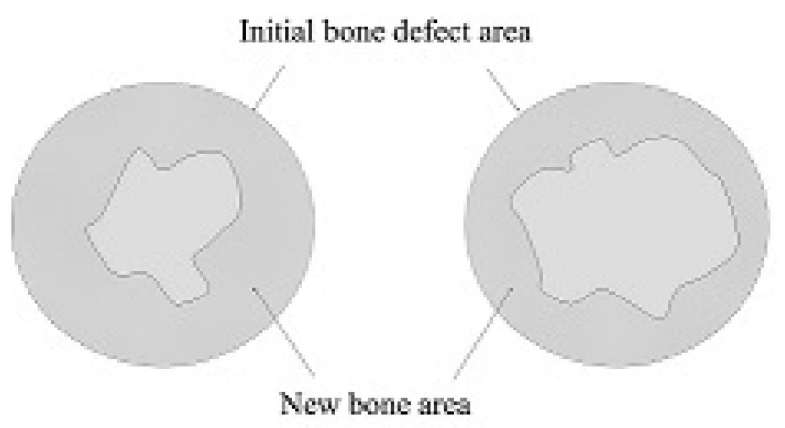

Figure2. The rate of new bone formation was defined as the space between the new bone area (shaded bydiagonallines) to the initial bone defect area(surrounded by dotted lines). The rate(\%) was measured using image analysis software.

of the left mandible under intravenous injection of Nembutal $(0.5$ $\mathrm{ml} / \mathrm{kg}$ weight), and the buccal alveolar mucosal surface in the distal portion of the most posterior tooth of the left mandible was incised diagonally forward, detached, and the right side of the mandible was exposed. A cylindrical defect in the bone (diameter $4.5 \mathrm{~mm}$ ) which passed through to the inside of the mandible (about $5 \mathrm{~mm}$ in depth) was formed posteriorly along the inferior border of the mandible with the anterior border of the masseteric insertion as a guide. Another bone defect was formed $3 \mathrm{~mm}$ in front of the other defect, therefore, forming two defects(Fig.1). $\beta$-TCP/CMchitin was implanted into the left posterior mandibular bone defect ( $\beta$-TCP/CM-chitin group), and the BONEJECT ${ }^{\mathrm{TM}}$ (KOKEN Co.

Ltd. Japan) that is a composite of sintered bovine bone granules and collagen gel, was implanted into the left anterior bone defect (HA group). Two new bone defects were made on the right side of the mandible in which $\beta$-TCP granules were implanted into the posterior bone defect ( $\beta$-TCP group) and no materials were implanted in the anterior bone defect (empty control group). The detached flap was put back in place and sutured. The number of test sites of each canine was four $(n=4)$, therefore there were a total of 64 bone defect sites in this study. Animals were euthanized by an overdose of ketamine hydrochloride at two weeks, one month, and three months after implantation. Lastly, the mandibular bone blocks containing the test pieces were harvested and stored in $10 \%$ neutral formalin solution for one week.

\section{Histological evaluation}

After bone blocks were soaked in decalcifying solution (PlankRychlo Method) for three days, they were embedded in paraffin. Tissue sections were cut out from each paraffin block in order to cover the whole bone defect area where the test pieces were implanted. After dissolution of paraffin, Hematoxylin-Eosin (HE) staining was carried out. HE-stained sections were observed using an optical microscope (E800, Nikon Corporation, Japan), and digital images of the histology were obtained. The newly formed 


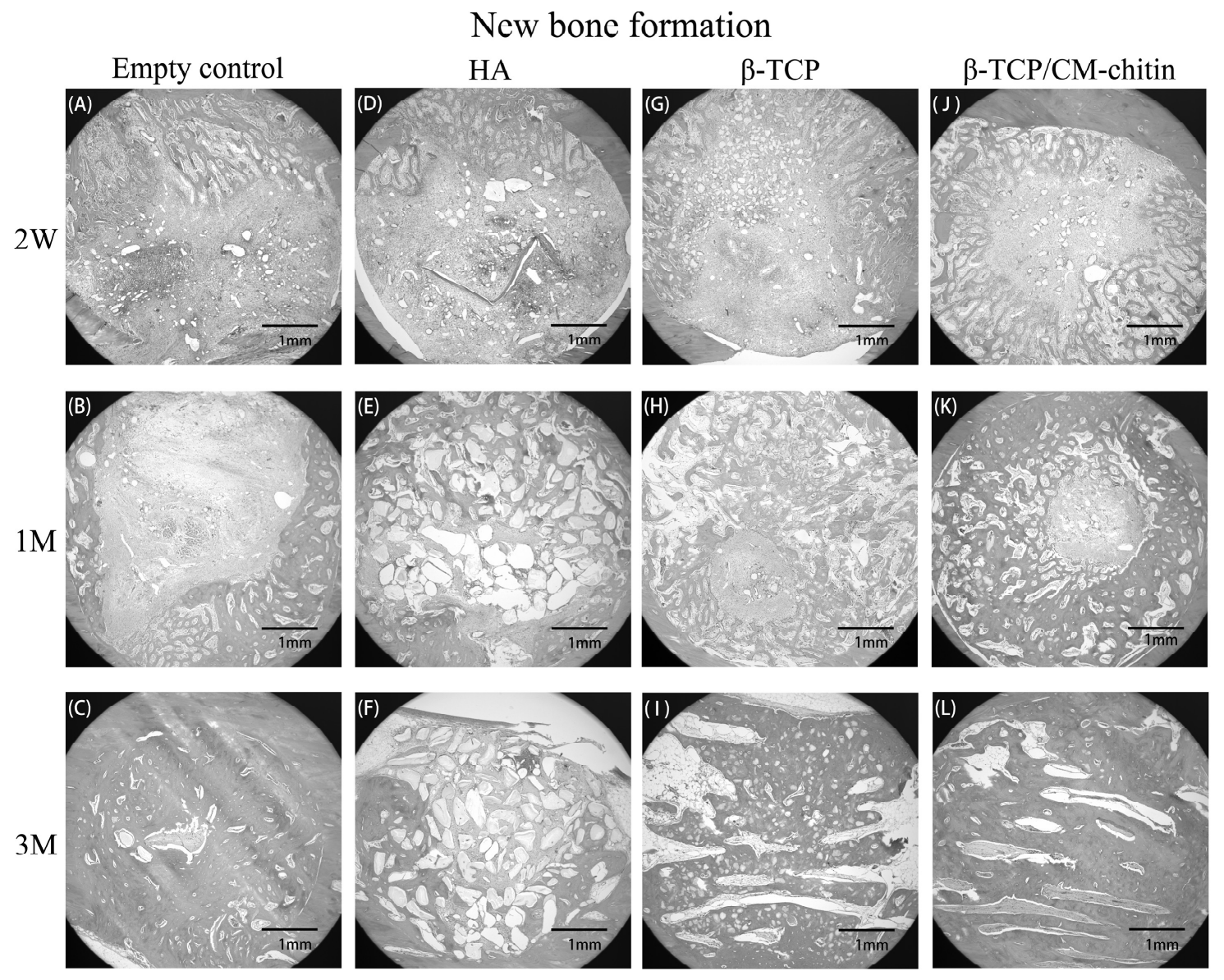

Figure 3. Histological cross sections of the control and bone substitutes at 2 weeks, 1 month, and 3 months after implantation. (A) Empty control 2 weeks after implantation; (B) Empty control 1 month after implantation; (C) Empty control 3 months after implantation; (D) HA substitute 2 weeks after implantation; (E) HA substitute 1 month after implantation; (F) HA substitute 3 months after implantation; (G) $\beta$-TCP substitute 2 weeks after implantation; (H) $\beta$-TCP substitute 1 month after implantation; (I) $\beta$-TCP substitute 3 months after implantation; (J) $\beta$-TCP /CM-chitin substitute 2 weeks after implantation; (K) $\beta$-TCP /CM-chitin substitute 1 month after implantation; (L) $\beta$-TCP /CM-chitin substitute 3 months after implantation. (HE staining, Bar $1 \mathrm{~mm}$ )

bone, in the bone defect area, that formed after implantation was measured using image analysis software (Spot Frex camera, Diagnostic Instruments Ltd., USA) (Fig. 2).

\section{Histological findings Empty control group}

At two weeks after surgery, most of the bone defects were occupied by fibrous connective tissue, but a small amount of immature new bone was seen on the surface of the pre-existing bone (Fig.3-A). Many capillaries and osteoblasts could be observed. At one month after surgery, new bone occupied about half of the initial bone defect area (Fig.3-B). At three months after surgery, new bone covered about $90 \%$ of the bone defect area and it showed advanced calcification (Fig.3-C, 4-A).

\section{HA group}

At two weeks after surgery, most of the bone defects were occupied by fibrous connective tissue and sintered bovine bone granules were distributed all across the bone defect. A small amount of immature new bone grew from the surrounding preexisting bone of the defected wall. Some bovine bone granules were surrounded by new bone (Fig.3-D). At one month, fibrous connective tissue was in the center of the bone defect and new bone occupied about the entire bone defect area. Many bovine bone granules were observed in the new bone (Fig.3-E). At three months, the new bone decreased to about $70 \%$ of the bone defect area and was replaced by fibrous connective tissue. The structure of new bone was mature and unevenly distributed on the surface of the bone defect. Sintered bovine bone granules remained in the original size both in the new bone and in the fibrous connective tissue, however, most of the granules were smooth (Fig.3-F, 4-B).

\section{$\beta$-TCP group}

At two weeks after surgery, most of the bone defects were occupied by fibrous connective tissue, and a small amount of immature new bone was observed on the bone defect wall (the pre-existing bone). Almost all the $\beta$-TCP granules were present 


\section{Magnified view of new bone formation at 3 months}

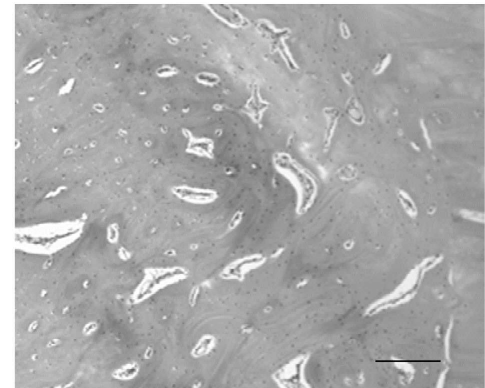

(A) Empty control

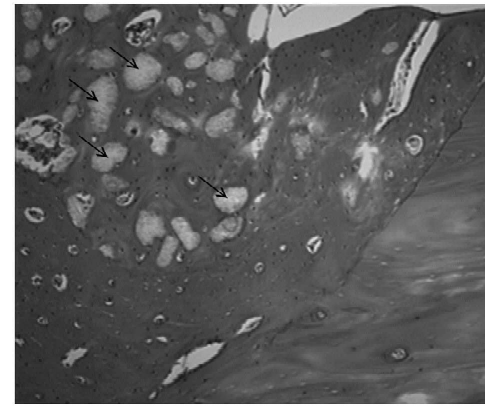

(C) $\beta$-TCP

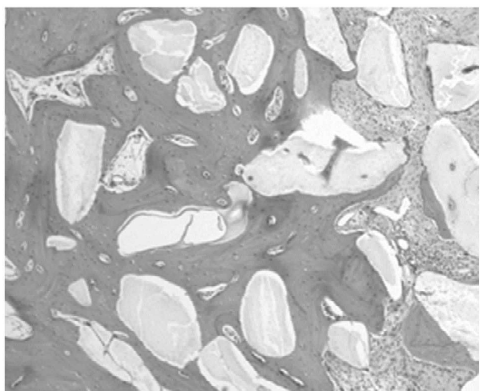

(B) HA

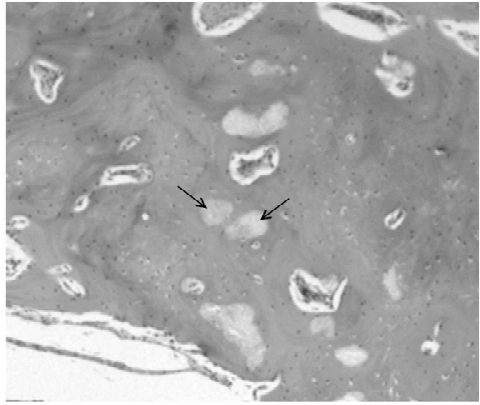

(D) $\beta-\mathrm{TCP} / \mathrm{CM}$-chitin

Figure 4. Histological cross section of the control and bone substitutes at 3 months. The empty control (A), $\beta$-TCP (C), and $\beta$-TCP/CM-chitin (D) showed similar amounts of new bone formation whereas HA (B) showed the least. Furthermore, the $\beta$-TCP/CM-chitin group showed less $\beta$-TCP granules remaining than the $\beta$-TCP group. (Arrows indicate areas of remaining $\beta$-TCP granules. HE staining; Bar $100 \mu \mathrm{m}$ )

in the fibrous connective tissue, and some were in the new bone (Fig.3-G). At one month after surgery, fibrous connective tissue was in the center of the bone defects and new bone occupied more than half of the defect area. Many $\beta$-TCP granules were seen in the new bone (Fig.3-H). At three months after surgery, new bone occupied the majority of the defect area. The new bone structure was mature and it was difficult to distinguish new bone from the surrounding pre-existing bone(Fig.3-I). Many $\beta$-TCP granules were observed in the new bone as well (Fig.4-C).

\section{$\beta-T C P / C M-c h i t i n$ group}

At two weeks after surgery, most of the bone defect was occupied by fibrous connective tissue. CM-chitin had already disappeared. $\beta$-TCP granules were distributed all across the bone defect, and a small number of $\beta$-TCP granules were observed in the new bone. New bone grew from the surrounding pre-existing bone (Fig.3-J). At one month after surgery, new bone occupied most of defect area and many $\beta$-TCP granules were seen in the new bone (Fig.3-K). At three months after surgery, new bone occupied almost all the areas of the bone defect. The bone structure was mature and it was difficult to distinguish between the new bone and the surrounding pre-existing bone(Fig.3-L). Most significantly, unlike the $\beta$-TCP group, few $\beta$-TCP granules were observed in the new bone (Fig.4-D).
The rate of new bone formation

$(\%)$

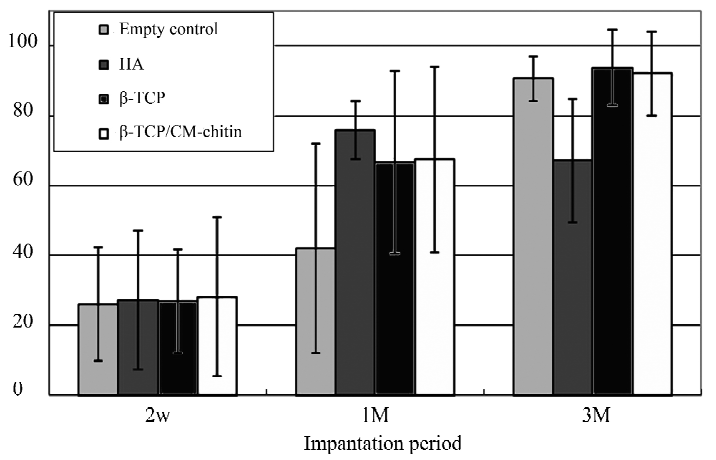

Figure 5. The rate of new bone formation was compared among the four test groups at intervals of 2 weeks, 1 month, and 3 months after implantation. The quantity of new bone of the $\beta$-TCP group and the $\beta$-TCP/CM-chitin group increased faster and more sufficiently than the empty control group and the HA group at the third month after implantation.

\section{Quantity of new bone formation}

The rate of new bone quantity in the cross section of the cortical bone defect was defined as the rate of new bone formation.Fig. 5 shows the rate of new bone formation compared among the four test groups at intervals of two weeks, one month, 
Daisuke Yamauchi et al::Bone Restoration ability of $\beta$-TCP and CM-Chitin

and three months after implantation. The quantity of new bone of the $\beta$-TCP group and the $\beta$-TCP/CM-chitin group increased faster and more sufficiently than the empty control group up to three months after implantation. Although the empty control group had less quantity of new bone compared with other groups up to one month, it became similar to the $\beta$-TCP group and the $\beta$-TCP/CMchitin group at three months. Although the quantity of new bone of the HA group was similar to the $\beta$-TCP group and the $\beta$-TCP/ $\mathrm{CM}$-chitin group up to one month, it was less than the other groups at three months.

The rate of new bone formation showed similar values in all groups at two weeks after implantation. The rate in the empty control group was $42.1 \%$ at one month, and $90.6 \%$ at three months. The rate in the HA group was $75.9 \%$ at one month, and $67.2 \%$ at three months. The rate in the $\beta$-TCP group was $66.6 \%$ at one month, and $93.8 \%$ at three months. The rate in the $\beta$-TCP/CMchitin group was $67.5 \%$ at one month, and $92.1 \%$ at three months. Statistical significant differences were not seen between the new bone formation ratein each group.

In the empty control group, the new bone area occupied more than $90 \%$ of the defect at three months after surgery. However, the amount of new bone formed was the least at month one after surgery out of the four groups. Yet, at three months after surgery, the amount of new bone formed was about the same in all groups. Therefore, it can be stated that the initial rate of new bone formation in the control group was slower than the other groups. The HA group showed similar rates of new bone formation to those of the $\beta$-TCP/CM-chitin group and the $\beta$-TCP group from two weeks until one month after surgery, but the rate of new bone formation decreased only in the HA group at after three months. The-TCP group showed steady growth of new bone formation over time, and the new bone area occupied more than $90 \%$ of the bone defect at three months after implantation. The $\beta-\mathrm{TCP} / \mathrm{CM}$-chitin group showed almost similar rates of new bone formation to those of the $\beta$-TCP group throughout the observed time periods.

\section{Discussion}

This study reported that $\beta$-TCP can stimulate bone formation at an early stage.However, little is known about the molecular mechanisms responsible for the beneficial effects of $\beta$-TCP in bone formation. Therefore, a separate series of experiments were previously conducted regarding the molecular mechanisms behind $\beta$-TCP using bone defects of canine mandibles. As part of those experiments, gene expression profiles were examined using DNA microarray. As a result, the study showed that connexin 43 expressed by $\beta$-TCP could play a role in osteogenesis through gap junctional intercellular bone-cell communication ${ }^{9}$. Furthermore, the study showed platelet-derived growth factor receptor beta protein expressed by $\beta$-TCP could be a mechanism to accelerate wound healing and bone formation ${ }^{10}$. Finally, the enhancement of HtrAl gene expressions in $\beta$-TCP implanted mandible bone was found to be a possible molecular mechanism for stimulating bone formation ${ }^{8}$. This experiment suggested that $\beta$-TCP enhances bone healing processes and stimulates the coordinated actions of osteoblasts and osteoclasts, leading to bone regeneration ${ }^{7}$.

The handling performances at the time of implantation were compared of each bone substitute. Handling performance of $\beta$ TCP granules gave mixed results; $\beta$-TCP granules spread to the periphery of the bone defect and were carried away by blood flow immediately after filling. Similarly, when sintered bovine bone granules in the HA group filled the bone defect, blood caused the granules to flow out of the bone defect in a short time. The $\beta$ $\mathrm{TCP} / \mathrm{CM}$-chitin was superior to these groups because it could easily be placed in the bone defect using tweezers. After placing it in the defect, blood infiltrated the spongy structure and maintained its position in the defect, as well as caused the granules in the structure to not spread. Furthermore, the volume of $\beta$-TCP granules comprised only about $30 \%$ of the $\beta$-TCP/CM-chitin structure, thus needing less $\beta$-TCP granules in the bone defect when utilizing it in the $\mathrm{CM}$-chitin structure ${ }^{19}$. Therefore, it was concluded that $\beta$-TCP/CM-chitin not only is easy to handle compared to solely granule bone substitute, but also a smaller amount of $\beta$-TCP can be used.

In this current study, new bone formation differed according to the type of material used and the time in which observations were made. At two weeks after surgery, the quantity of new bone was similar in all four groups. However thereafter, in the control group, there was a gradual increase of new bone in the first month and it occupied more than $90 \%$ of the defect in the third month. The HA group showed similar rates of new bone formation to those of the $\beta$-TCP/CM-chitin group and the $\beta$-TCP group from two weeks until one month, but the rate of new bone formation decreased only in this group three months after implantation. In the $\beta$-TCP group, quantity of new bone was larger than in the control group in the first month, yet, it reached similar levels in the third month.

The $\beta$-TCP/CM-chitin group differed from the other groups as several significant advantages were noted: First, the $\beta$-TCP/ $\mathrm{CM}$-chitin group showed equivalent patterns of new bone growth and quantity as the $\beta$-TCP group, therefore, confirming that the use of CM-chitin did not interfere with new bone formation. Various cells derived from the bone marrow, which plays an important role in organizing restorations, could easily infiltrate the sponge structure of the $\beta$-TCP/CM-chitin composite, allowing the composite to function as a scaffold and facilitate bone restoration.In contrast, in the HA group, the center of the bone defect was filled with fibrous connective tissue and mature bone structure was observed only on the periphery of the bone defect at three months after implantation. Sintered bovine bone granules 
containing HA were present at the center of the bone defect, but most were surrounded by fibrous connective tissue. In another in vivo study,when HA was implanted in a canine bone defect, the phenomenon in which the new bone gradually disappeared was also observed ${ }^{20)}$.

Secondly, as seen in a previously conducted study, $\beta$-TCP/ CM-chitin showed better handling performances than the other groups. It could easily be placed in the bone defect using tweezers and the $\beta$-TCP granules were fixated in the bone defect as the sponge structure of the CM-chitin absorbed the flow of blood. In contrast, when administering HA in the bone defect, a syringe was prepared with HA sintered bovine bone granules in a collagen gel, as it is easier to transfer and inject the granules into the bone defect. However, when the granules filled the bone defect, blood caused the gelto flow out of the bone defect. Additionally, as each unit of the $\beta$-TCP/CM-chitin are larger in size, recording the amount of $\beta$-TCP/CM-chitin used in one bone defect was also easier than measuring the amount of $\beta$-TCP granules.

Thirdly, although the new bone formation was similar in the $\beta$-TCP group and the $\beta$-TCP/CM-chitin group, the quantity of $\beta$ TCP granules used in the $\beta$-TCP/CM-chitin group was less than in the $\beta$-TCP group due to its three dimensional matrix. Therefore, less unabsorbed $\beta$-TCPgranules remained when new bone was formed, leading to less risk of infections and other complications.

The study showed that the $\beta$-TCP/CM-chitin group demonstrated a similar ability to promote bone formation as the $\beta$-TCP group, and exhibited better handling performances than other granular forms of bone substitutes. Therefore it can be concluded that $\beta$-TCP/CM-chitin could be used as a suitable filler for bone regeneration in oral surgery.

\section{References}

1. Mathai JK, Chandra S, Nair KV and Nambiar KK. Tricalcium phosphate ceramic as immediate root implants for the maintenance of alveolar bone in partially edentulous mandibular jaws. A clinical study. Aust Dent J 34: 421-6, 1989

2. Chung HJ andChung EH. Tricalcium phosphate implant in periodontal defects - initial 3 months Article in Korean. TaehanChikkwaUisaHyophoe Chi 27: 155-9, 1989

3. Zaffe D, Gatti AM, Poli GP, Ravaglioli A and Krajewski A. Comparative analyses among interfaces of some ceramic materials and bone in sheep. Bull Group Int Rech SciStomatol Odontol 33: 95-100, 1990

4. Trisi P, Rao W, Rebaudi A andFiore P. Histologic effect of pure-phase beta-tricalcium phosphate on bone regeneration in human artificial jaw bone defects. Int J Perio Rest Dent 23: 69-77, 2003

5. Metsger DS, Driskell TD and Paulsrud JR. Tricalcium phosphate ceramic - Aresorbable bone implant: Review and current status. J Am Dent Assoc 105: 1035-8, 1982

6. Yoshitake K, Takahashi T, Hayashi M, Watanabe T, Zhao J and Abiko Y.Fibronectin and focal adhesion kinase gene expression in dog mandible implanted by $\beta$-TCP. J Oral Tissue Engin 8: 1-8, 2010

7. Zhao J, Watanabe T, Bhawal UK, Kubota E and Abiko Y. Transcriptome analysis of $\beta$-TCP implanted in dog mandible. Bone 48: 864-77, 2011

8. Watanabe T, Zhao J, Hayashi M, Huang MY, Imai K, Takahashi T and Abiko Y. High-temperature requirement protein A1 (HtrA1) gene expression in dog mandible bone by $\beta$-TCP. J Hard Tissue Biol 19: 23-26, 2010

9. Hayashi M, Takahashi T, Kawaguchi K, Watanabe T, Zhao J and Abiko Y. Connexin 43 expression at an early stage in dog mandibles by $\beta$-TCP. Dent Mater J 30: 58-65, 2011

10. Chu CH, Oshitani T, Takahashi T, Zhao J, Lee SY, Watanabe $\mathrm{T}$ and Abiko Y.Expression of platelet-derived growth factor receptor beta in dog mandible by beta-tricalcium phosphate. Int J Oral-Med Sci 9: 190-196, 2011

11. Okamoto Y, Minami S, Matsuhashi A, Sashiwa H, Saimoto H, Shigemasa Y, Tanigawa T, Tanaka Y andTokura S. Polymeric N-acetyl-D-glucosamine (chitin) induces histionic activation in dogs. J Vet Med Sci 55: 739-42, 1993

12. Takiguchi H, Koga D and Taihou A. Chitin, Chitosan Hand Book, Gihodo Shuppan Co., Ltd., Tokyo, 1995.

13. Muramatsu K, Nakajima M, Kikuchi M, Shimada S, Sasaki $\mathrm{K}$, Masuda S and Yoshihara Y. In vitro cytocompatibility assessment of beta-tricalcium phosphate/carboxymethylchitin composite. J Biomed Mater Res 15: 635-643, 2004

14. Jarmila V and Vavríková E. Chitosan derivatives with antimicrobial, antitumour and antioxidant activities- A review. Curr Pharm Des 17: 3596-607, 2011

15. Jayakumar R, Chennazhi KP, Srinivasan S, Nair SV, Furuike $\mathrm{T}$ and Tamura $\mathrm{H}$. Chitin scaffolds in tissue engineering. Int $\mathrm{J}$ Mol Sci 12: 1876-87, 2011

16. Jayakumar R, Prabaharan M, Nair SV and Tamura H. Novel chitin and chitosan nanofibers in biomedical applications. Bio Technol Adv 28: 142-50, 2010

17. Taniyama K, Shirakata Y, Yoshimoto T, Takeuchi N, Yoshihara $\mathrm{Y}$ and Noguchi $\mathrm{K}$. Bone formation using $\beta$-tricalcium phosphate/carboxymethyl-chitin composite scaffold in rat calvarial defects. Oral Surg Oral Med Oral Pathol Oral Radiol2:281-287,2012

18. Muramatsu K, Oba K, Mukai D, Hasegawa K, Masuda S and Yoshihara Y. Subacute systemic toxicity assessment of betatricalcium phosphate/carboxymethyl-chitin composite implanted in rat femur. J Mater Sci Mater Med 18: 513-22, 2007

19. Liao F, Chen Y, Li Z, Wang Y, Shi B, Gong Z and Cheng XJ. A novel bioactive three-dimensional beta-tricalcium 
Daisuke Yamauchi et al.:Bone Restoration ability of $\beta$-TCP and CM-Chitin

phosphate/chitosan scaffold for periodontal tissue engineering. J Mater Sci Mater Med 21: 489-96, 2010

20. Tabata M, Shimoda T, Sugihara K, Ogomi D, Serizawa T and Akashi M. Osteoconductive and hemostatic properties of apatite formed on/in agarose gel as a one-grafting material. J Biomed Mater Res B Appl Bio Mater 67: 680-8, 2003 
J.Hard Tissue Biology Vol. 22(4):473-480, 2013 